\title{
Supporting Information \\ Solvent Mediated Excited State Proton Transfer in \\ Indigo Carmine
}

Partha Pratim Roy ${ }^{1,2}$, James She ${ }^{3}$, Eric A. Arsenault ${ }^{1,2,4}$, Yusuke Yoneda ${ }^{1,2,4}$, Katelyn

Feuling ${ }^{1,2,5}$, Martin Head-Gordon ${ }^{3,4}$, and Graham R. Fleming ${ }^{1,2,4 *}$

${ }^{1}$ Department of Chemistry, University of California, Berkeley, CA 94720, USA.

${ }^{2}$ Molecular Biophysics and Integrated Bioimaging Division, Lawrence Berkeley National Laboratory, Berkeley, CA 94720, USA.

${ }^{3}$ Department of Chemistry, Kenneth S. Pitzer Center for Theoretical Chemistry, University of California, Berkeley, CA 94720, USA.

${ }^{4}$ Kavli Energy Nanoscience Institute at Berkeley, Berkeley, CA 94720, USA.

${ }^{5}$ Department of Chemistry, University of Minnesota, Minneapolis, MN 55455, USA.

AUTHOR INFORMATION

Corresponding Author

*grfleming@lbl.gov 


\section{Section I: Experimental data}

Experimental details: The pump-probe and $2 \mathrm{DEV}$ experimental apparatus with a partially collinear pump-probe geometry used for this study has been explained in detail previously. ${ }^{1}$ In brief, the visible excitation spectrum (pulse duration: $10 \mathrm{fs}$, energy: $200 \mathrm{~nJ}$ ) was centered around the $\mathrm{S}_{0} \rightarrow \mathrm{S}_{1}$ absorption maxima of InC (Fig. S1). The pulse duration was measured by autocorrelation based on two photon absorption in a SiC photodiode. To determine the IRF (and from this estimate the IR pulse duration), a cross correlation between the IR and visible pulses in a 50 micron Ge plate was performed. The infrared probe spectrum with a pulse duration of $\sim 70 \mathrm{fs}$ covered the fingerprint frequency region from 1490 to $1660 \mathrm{~cm}^{-1}$. For 2DEV, a pulse shaper (Dazzler, Fastlite) was utilized to derive an excitation pulse pair and to control the phase and delay between them. For each waiting time, a 2D spectrum was collected as a function of coherence time (scanned for 100 fs with 2.5 fs time step) and detected via a spectrograph (Horiba Triax) coupled with a dual-array 64 element $\mathrm{HgCdTe}$ detector. At each $\tau$ delay (coherence time),

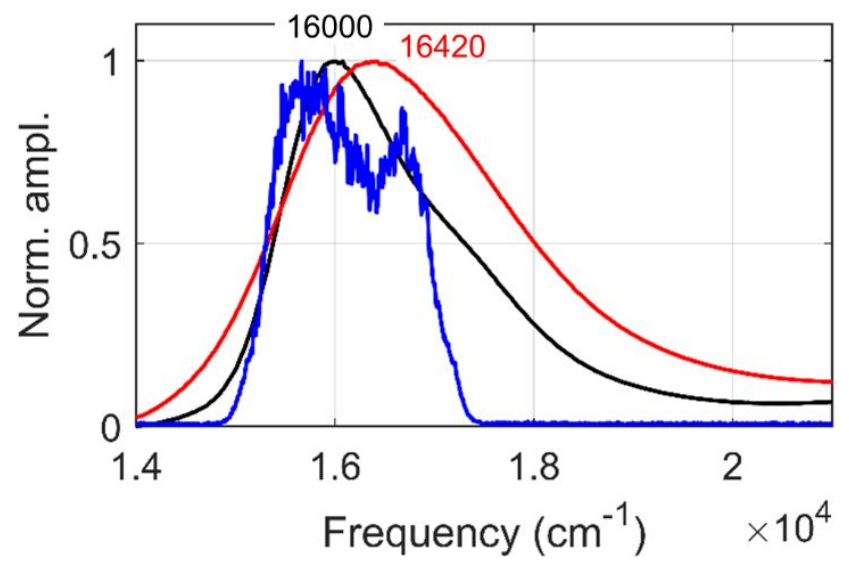

Figure S1. The normalized UV-Vis linear absorption spectra of InC in dDMSO (black) and $\mathrm{D}_{2} \mathrm{O}$ (red). The blue curve represents the excitation spectrum used in pump-probe and 2DEV experiments. 
the signal was measured with the relative phase, $\varphi_{12}$, set to $0,2 \pi / 3,4 \pi / 3$ and a $3 \times 1 \times 1$ phase cycling scheme was employed to remove the pump-probe background signal. The signal was collected in the fully rotated frame with respect to $\tau$ in order to remove the optical frequency of the pump laser. The waiting time $(T)$ was scanned by a motor-controlled delay stage. The coherence time axis was Fourier transformed to obtain the $2 \mathrm{DEV}$ frequency-frequency correlation map (Fig. 3). All the measurements were carried out with the sample concentration of $0.7 \mathrm{OD}$ at $\lambda_{\max }$ in a flow cell with 0.25 and $0.1 \mathrm{~mm}$ optical path lengths for InC in dDMSO and $\mathrm{D}_{2} \mathrm{O}$, respectively. Shorter path length was used to measure in $\mathrm{D}_{2} \mathrm{O}$ to minimize absorption of IR probe by $\mathrm{D}_{2} \mathrm{O}$.

Exponential fitting of the pump-probe transients: The pump-probe transients for different vibrational bands (Fig. 1b, c) of $\mathrm{InC}$ in dDMSO and $\mathrm{D}_{2} \mathrm{O}$ are shown Fig. S2 and S3-S4, respectively. In each graph, the black circles represent experimental data points and solid blue line represents the exponential fit. The initial $200 \mathrm{fs}$ of each transient were excluded from the fitting to avoid the coherent artifact.
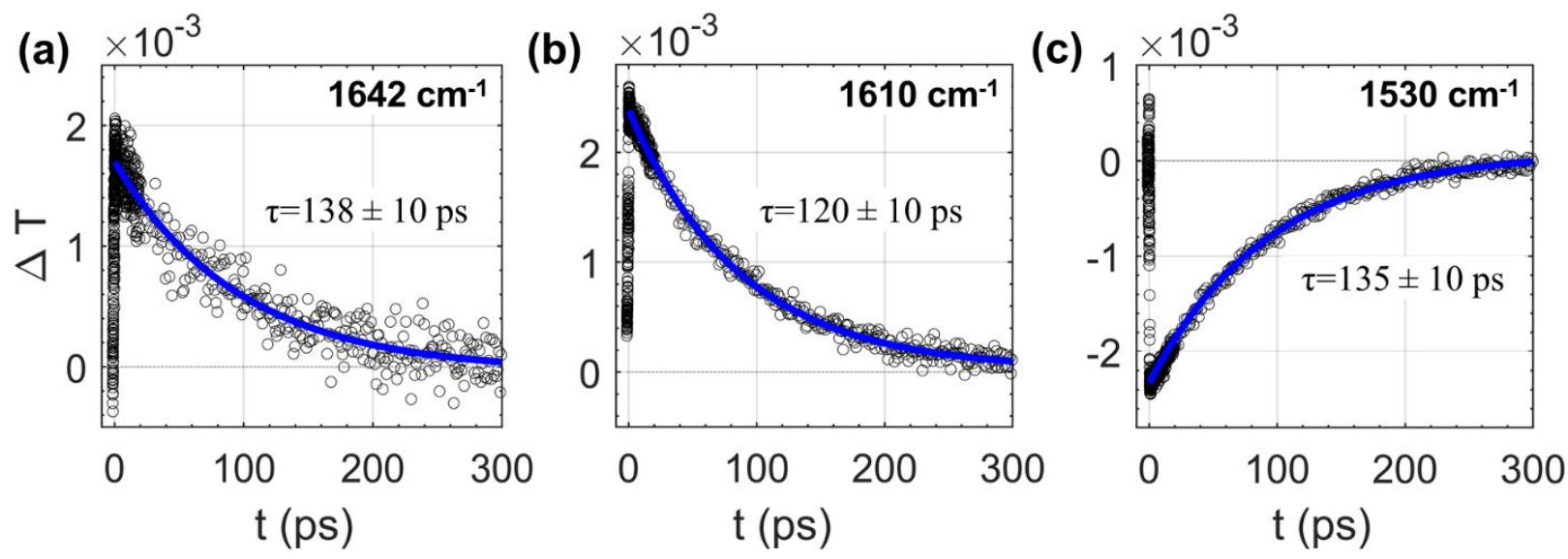
Figure S2. Mono-exponential fitting of the transients obtained in pump-probe experiments of InC in dDMSO at detection frequencies of (a) $1642 \mathrm{~cm}^{-1}$, (b) $1610 \mathrm{~cm}^{-1}$ and (c) $1530 \mathrm{~cm}^{-1}$.
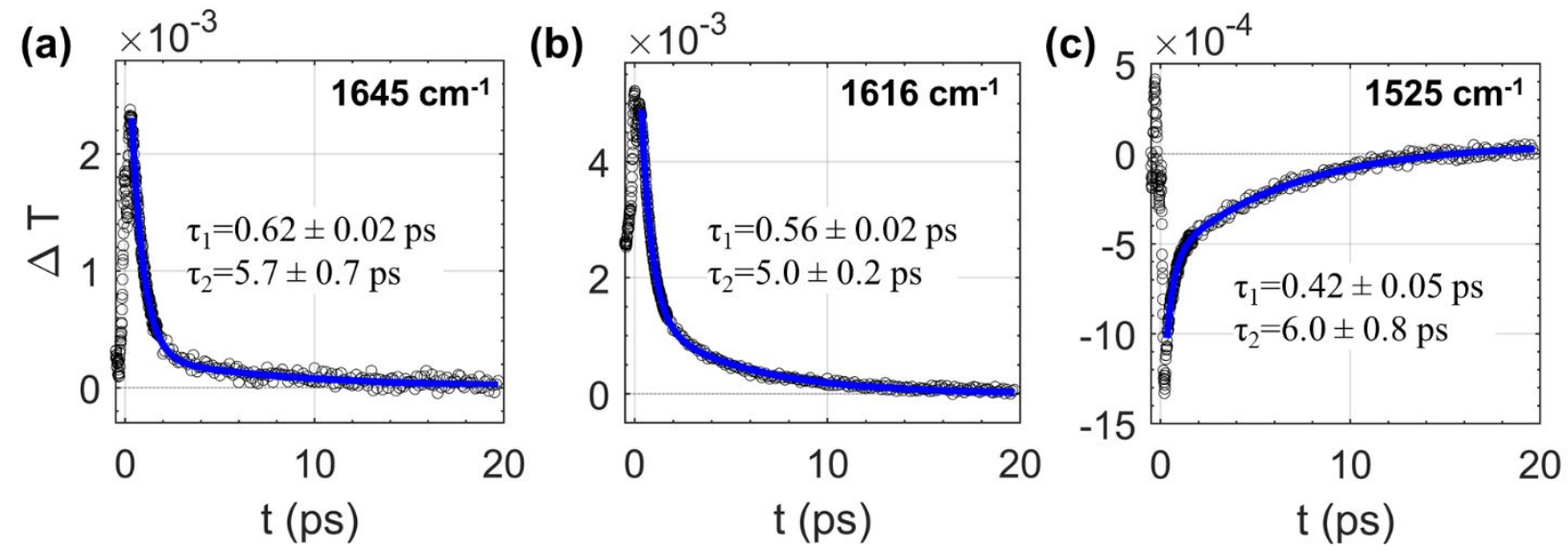

Figure S3. Bi-exponential fitting of the transient obtained in pump-probe experiments of InC in $\mathrm{D}_{2} \mathrm{O}$ at detection frequency of (a) $1645 \mathrm{~cm}^{-1}$, (b) $1616 \mathrm{~cm}^{-1}$ and (c) $1525 \mathrm{~cm}^{-1}$.

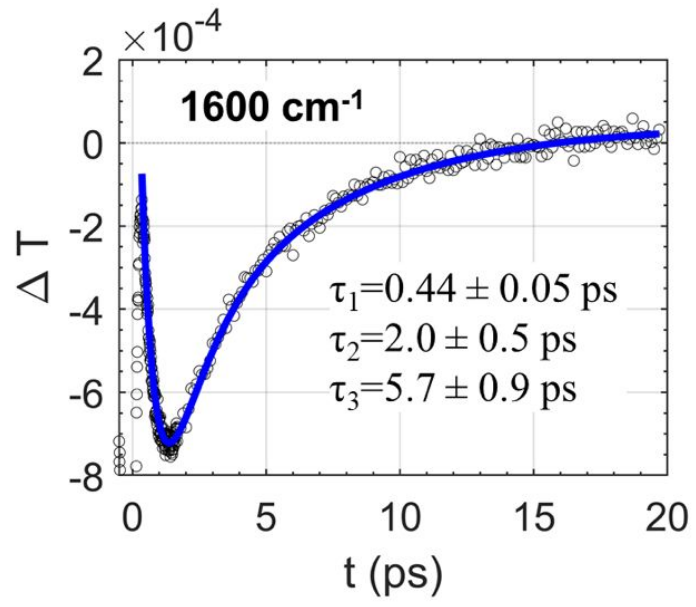


Figure S4. Tri-exponential fitting of the transient obtained in pump-probe experiments of InC in $\mathrm{D}_{2} \mathrm{O}$ at detection frequency of $1600 \mathrm{~cm}^{-1}$. It shows an exponential rise followed by a biexponential decay. 


\section{Section II: Theoretical calculations}

Regarding the vibrational frequency calculations, we utilize a scaling factor of 0.96 throughout.

Table S1. TDDFT calculation of Indigo with $6-31+\mathrm{G}^{*}$ basis set at B3LYP level of theory.

\begin{tabular}{|c|c|c|}
\hline Indigo & Total energy $[\mathrm{Ha}]$ & $\Delta \mathrm{E}(\mathrm{eV})$ \\
\hline keto $\mathrm{S}_{0}$ min & -875.7473 & - \\
\hline keto $\mathrm{S}_{1} \mathrm{FC}$ & -875.6722 & 2.04 \\
\hline keto $\mathrm{S}_{1} \min$ & -875.6816 & -0.26 \\
\hline enol $\mathrm{S}_{1} \min$ & -875.6706 & 0.30 \\
\hline
\end{tabular}

Table S2. TDDFT calculation of Indigo Carmine with $6-31+\mathrm{G}^{*}$ basis set at B3LYP level of theory.

\begin{tabular}{|c|c|c|}
\hline Indigo Carmine & Total energy $[\mathrm{Ha}]$ & $\Delta \mathrm{E}(\mathrm{eV})$ \\
\hline keto $\mathrm{S}_{0} \mathrm{~min}$ & -2446.9650 & - \\
\hline keto $\mathrm{S}_{1} \mathrm{FC}$ & -2446.8875 & 2.11 \\
\hline keto $\mathrm{S}_{1} \mathrm{~min}$ & -2446.8968 & -0.25 \\
\hline enol $\mathrm{S}_{1} \mathrm{~min}$ & -2446.8859 & 0.30 \\
\hline
\end{tabular}




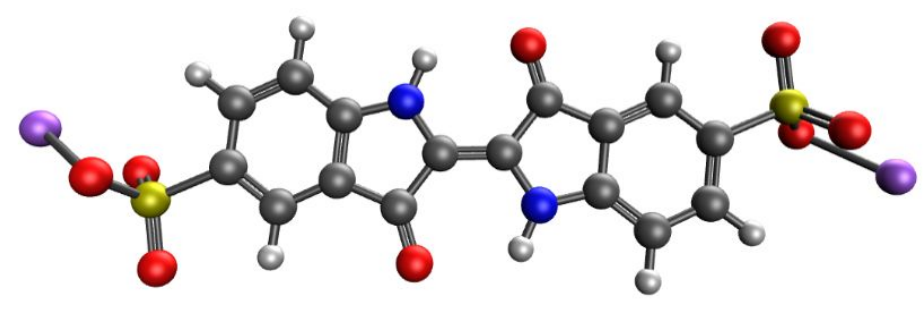

Figure S5. The optimized geometry of keto- $\mathrm{S}_{1}$ of InC calculated at the TDDFT/B3LYP/6-31+G* level of theory. The geometry is very similar to that of the keto- $\mathrm{S}_{0}$ minimum shown in Fig. $1 \mathrm{~d}$ of the main text.

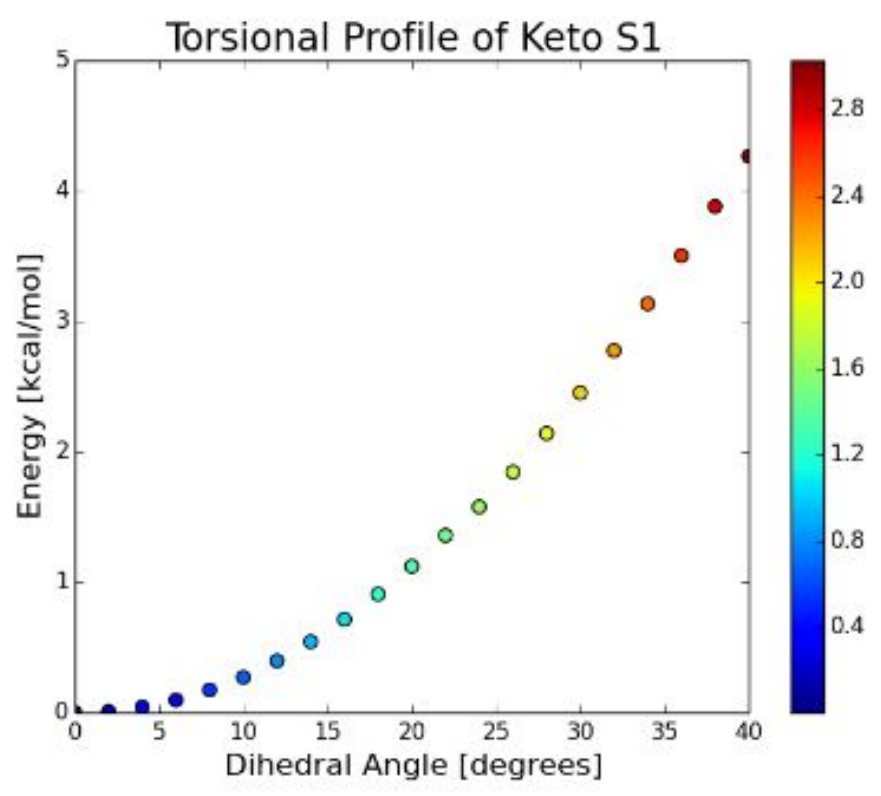

Figure S6. The calculated energy relative to the planar configuration (dihedral angle $=0$ degrees) of the keto- $\mathrm{S}_{1}$ minimum as a function of dihedral angle around the central $\mathrm{C}=\mathrm{C}$ bond in Indigo. The corresponding dipole moments are shown via the color scale on the right. TDDFT/B3LYP/6-31+G* level of theory. 

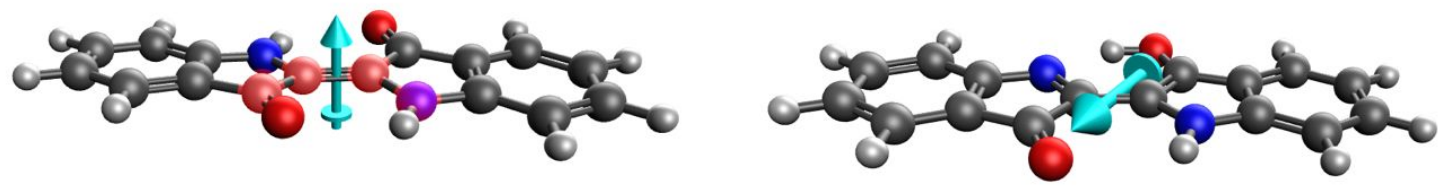

Figure S7. Dipole moments, calculated at the TDDFT/B3LYP/6-31+G* level of theory, overlaid on (left) the keto-S $\mathrm{S}_{1}$ minimum geometry for Indigo, optimized with a 14 degrees dihedral constraint involving the $\mathrm{N}-\mathrm{C}-\mathrm{C}-\mathrm{C}$ atoms highlighted in red, and (right) enol- $\mathrm{S}_{1}$ minimum. As given by excited-state geometry optimization calculations, the dipole moment vectors of the twisted keto- $\mathrm{S}_{1}$ state and enol-S $\mathrm{S}_{1}$ minimum are $(0.87,0.02,0.00)$ and $(0.97,1.54,-0.69)$, with magnitudes of 0.94 and 1.94 Debyes, respectively. We argue in the main text that this significant change in direction and increase in magnitude is consistent with the change in sign of the CLS.
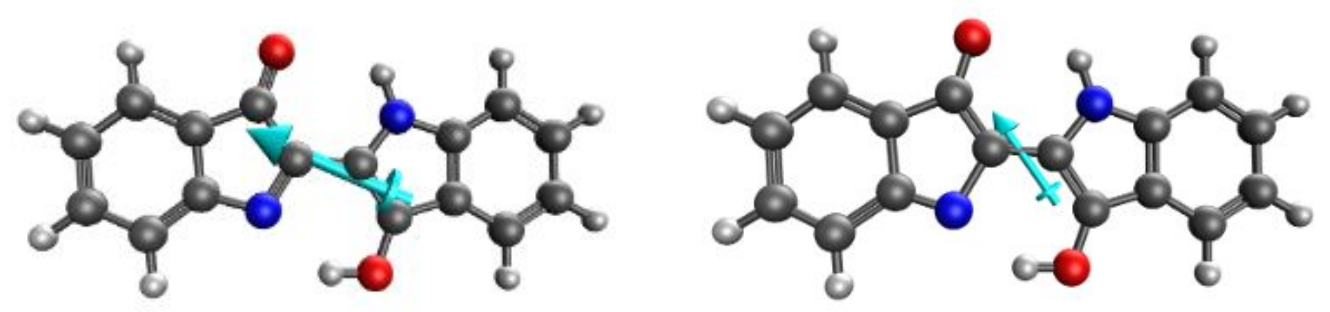

Figure S8. Dipole moments overlaid on (left) the enol- $\mathrm{S}_{1}$ minimum geometry for Indigo, and (right) the $\mathrm{S}_{1} / \mathrm{S}_{0}$ MECP geometry. These calculations utilized the SF-TDDFT/BHHLYP/6$31+\mathrm{G}^{*}$ level of theory. As given by excited-state geometry optimization calculations, the dipole moment vectors of the enol- $\mathrm{S}_{1}$ minimum and MECP are $(-0.22,-1.80,4.57)$ and $(-0.11,-1.11$, 0.83), with magnitudes of 4.92 and 1.39 Debyes, respectively. 
The Cartesian coordinates of the optimized structures are tabulated below.

Indigo Carmine, keto $\mathrm{S}_{0}$ :

Coordinates (Angstroms)

\begin{tabular}{|c|c|c|c|}
\hline ATOM & $X$ & $\mathrm{Y}$ & \\
\hline $1 \mathrm{~N}$ & 0.4890203403 & -1.6016022994 & 1.6232438966 \\
\hline $2 \mathrm{C}$ & -0.7099780820 & -2.0300564776 & 2.1609223117 \\
\hline $3 \mathrm{C}$ & 0.2596225358 & -0.8388016839 & 0.4962180200 \\
\hline $4 \mathrm{C}$ & -1.7877672947 & -1.5500600794 & 1.3793116383 \\
\hline $5 \mathrm{C}$ & -1.2174386923 & -0.7683183335 & 0.2799239324 \\
\hline $6 \mathrm{C}$ & 1.2139889500 & -0.2512637133 & -0.2837879587 \\
\hline $7 \mathrm{C}$ & 2.6910317200 & -0.3212521017 & -0.0671299728 \\
\hline $8 \mathrm{C}$ & 3.2613399780 & 0.4611479443 & -1.1660733451 \\
\hline $9 \mathrm{C}$ & 2.1835651885 & 0.9409439050 & -1.9478367879 \\
\hline $10 \mathrm{~N}$ & 0.9846189633 & 0.5113682836 & -1.4109238776 \\
\hline 110 & -1.7728818237 & -0.1635039262 & -0.6464270557 \\
\hline $12 \mathrm{O}$ & 3.2464634071 & -0.9260456413 & 0.8592254373 \\
\hline $13 \mathrm{C}$ & -0.9329142982 & -2.8177456655 & 3.2960838633 \\
\hline $14 \mathrm{C}$ & -2.2528053261 & -3.1092524665 & 3.6278266495 \\
\hline $15 \mathrm{C}$ & -3.3288524728 & -2.6300038448 & 2.8490176566 \\
\hline $16 \mathrm{C}$ & -3.1094357404 & -1.8471277767 & 1.7186201426 \\
\hline $17 \mathrm{C}$ & 4.5829675536 & 0.7589542939 & -1.5048368534 \\
\hline $18 \mathrm{C}$ & 4.8023843286 & 1.5424425694 & -2.6348299751 \\
\hline $19 \mathrm{C}$ & 3.7263693956 & 2.0215428221 & -3.4137369582 \\
\hline $20 \mathrm{C}$ & 2.4064962777 & 1.7292331304 & -3.0825670926 \\
\hline $21 \mathrm{H}$ & 1.4135614065 & -1.8005134983 & 1.9888344531 \\
\hline $\mathrm{H}$ & 0.0599996933 & 0.7117048730 & -1.7755407716 \\
\hline
\end{tabular}




$\begin{array}{lrrrr}23 \mathrm{H} & -0.1100431820 & -3.1899588505 & 3.8984338246 \\ 24 \mathrm{H} & -3.9378105336 & -1.4810438601 & 1.1212951139 \\ 25 \mathrm{H} & 5.4113311549 & 0.3929856484 & -0.9074287624 \\ 26 \mathrm{H} & 1.5836639401 & 2.1013209516 & -3.6850470012 \\ 27 \mathrm{H} & -2.4481491265 & -3.7201649490 & 4.5041088191 \\ 28 \mathrm{H} & 3.9216702213 & 2.6329578798 & -4.2896804835 \\ 29 \mathrm{~S} & 6.4833367923 & 1.9463316767 & -3.1184113401 \\ 30 \mathrm{O} & 6.6981088867 & 1.3767143231 & -4.5039801025 \\ 31 \mathrm{O} & 6.5626189998 & 3.4529285343 & -3.2319419143 \\ 32 \mathrm{O} & 7.3802122145 & 1.3711864117 & -2.0874306694 \\ 33 \mathrm{~S} & -5.0098008855 & -3.0328268877 & 3.3334444032 \\ 34 \mathrm{O} & -5.0897154087 & -4.5393251737 & 3.4479569276 \\ 35 \mathrm{O} & -5.2238390598 & -2.4623161870 & 4.7187414033 \\ 36 \mathrm{O} & -5.9067997483 & -2.4579966736 & 2.3023969526 \\ 37 \mathrm{Na} & 7.1683641768 & 3.4097373932 & -5.4432413371 \\ 38 \mathrm{Na} & -5.6945516497 & -4.4956283506 & 5.6588676137\end{array}$

Indigo Carmine, keto $\mathrm{S}_{1}$ :

Coordinates (Angstroms)

$\begin{array}{ccccc}\text { ATOM } & \mathrm{X} & \mathrm{Y} & \mathrm{Z} & \\ 1 \mathrm{~N} & 0.4588522483 & -1.5930645231 & 1.6023850907 \\ 2 \mathrm{C} & -0.7260760684 & -2.0323791798 & 2.1528963933 \\ 3 \mathrm{C} & 0.2248979122 & -0.8313502756 & 0.4827657360 \\ 4 \mathrm{C} & -1.8008063741 & -1.5419086472 & 1.3616746482 \\ 5 \mathrm{C} & -1.2254382254 & -0.7560163567 & 0.2627549943 \\ \text { 6 C } & 1.2487766763 & -0.2569032272 & -0.2688925799 \\ 7 \mathrm{C} & 2.6991115971 & -0.3321703756 & -0.0489003470 \\ \text { 8 C } & 3.2744409431 & 0.4537759468 & -1.1478120756\end{array}$




\begin{tabular}{lllll}
$9 \mathrm{C}$ & 2.1996849022 & 0.9441702128 & -1.9390324220 \\
$10 \mathrm{~N}$ & 1.0147772323 & 0.5047822232 & -1.3885213033 \\
$11 \mathrm{O}$ & -1.7983802143 & -0.1441701226 & -0.6703990398 \\
$12 \mathrm{O}$ & 3.2721031418 & -0.9439857468 & 0.8842405795 \\
$13 \mathrm{C}$ & -0.9334497863 & -2.8223356455 & 3.2855444341 \\
$14 \mathrm{C}$ & -2.2546656800 & -3.1214660081 & 3.6274308341 \\
$15 \mathrm{C}$ & -3.3233232220 & -2.6371932877 & 2.8482499235 \\
$16 \mathrm{C}$ & -3.1138448996 & -1.8454518620 & 1.7107460287 \\
$17 \mathrm{C}$ & 4.5874607933 & 0.7574049169 & -1.4968836270 \\
$18 \mathrm{C}$ & 4.7968823916 & 1.5491744437 & -2.6343798020 \\
$19 \mathrm{C}$ & 3.7281954696 & 2.0333736935 & -3.4135520482 \\
$20 \mathrm{C}$ & 2.4069974369 & 1.7341491008 & -3.0716725310 \\
$21 \mathrm{H}$ & 1.3959253936 & -1.7848999953 & 1.9530353599 \\
$22 \mathrm{H}$ & 0.0776814077 & 0.6965530690 & -1.7391596698 \\
$23 \mathrm{H}$ & -0.1032446278 & -3.1918336040 & 3.8797413264 \\
$24 \mathrm{H}$ & -3.9525252377 & -1.4847981740 & 1.1246758586 \\
$25 \mathrm{H}$ & 5.4261658444 & 0.3968033627 & -0.9108176340 \\
$26 \mathrm{H}$ & 1.5767661644 & 2.1035962139 & -3.6658636968 \\
$27 \mathrm{H}$ & -2.4472774668 & -3.7353546050 & 4.5013333525 \\
$28 \mathrm{H}$ & 3.9207645141 & 2.6472812919 & -4.2874510040 \\
$29 \mathrm{~S}$ & 6.4825499517 & 1.9506735816 & -3.1199868820 \\
$30 \mathrm{O}$ & 6.6968072762 & 1.3593470888 & -4.4954129564 \\
$31 \mathrm{O}$ & 6.5533747499 & 3.4547098930 & -3.2548442550 \\
$32 \mathrm{O}$ & 7.3773129108 & 1.3933153032 & -2.0787229553 \\
$33 \mathrm{~S}$ & -5.0090167177 & -3.0385672859 & 3.3338551851 \\
$34 \mathrm{O}$ & -5.0799233642 & -4.5425893951 & 3.4688141208 \\
$35 \mathrm{O}$ & -5.2232711996 & -2.4471347296 & 4.7092390244 \\
36 & -5.9037372803 & -2.4812305815 & 2.2925417990 \\
\hline 1.1535622651 & 3.3826609298 & -5.4701794996 \\
\hline
\end{tabular}


$\begin{array}{llll}38 \mathrm{Na} & -5.6801580582 & -4.4702754432 & 5.6841804397\end{array}$

Indigo Carmine, enol $\mathrm{S}_{1}$ :

Coordinates (Angstroms)

\begin{tabular}{|c|c|c|c|}
\hline $\mathrm{rO}$ & $\mathrm{X}$ & Y & \\
\hline $1 \mathrm{~N}$ & 3491793109 & 0.7242312602 & .37516627 \\
\hline $2 \mathrm{C}$ & 25947 & 331752375 & 35074067 \\
\hline $3 \mathrm{C}$ & 0.9007991113 & 1.2346426269 & 1.1878027541 \\
\hline $4 \mathrm{C}$ & 3677 & 2.2704768991 & 8992 \\
\hline $5 \mathrm{C}$ & -0.1635628404 & 2.2270704256 & 1.3655650685 \\
\hline $5 \mathrm{C}$ & 1.4840114867 & 0.7738342909 & -0.0092336079 \\
\hline $\mathrm{C}$ & 2.5221843256 & -0.1970724150 & -0.121 \\
\hline $\mathrm{C}$ & 2.8113108307 & -0.3575376602 & 6637 \\
\hline $9 \mathrm{C}$ & 1.9327713462 & 0.5267300747 & -2.201 \\
\hline $\mathrm{N}$ & 1.1520829350 & 1.1870022872 & -1.2801971926 \\
\hline 10 & -0.7659446845 & 2.8631763553 & 0.4644550161 \\
\hline $2 \mathrm{C}$ & 0.7408800010 & 1.1249126947 & 25566 \\
\hline $3 \mathrm{C}$ & -0. & 6 & 13 \\
\hline $4 \mathrm{C}$ & -1.0245614856 & 2.7710168845 & 5.0532105979 \\
\hline $5 \mathrm{C}$ & -1.1517938767 & 2.9921951719 & 3.6707084411 \\
\hline 5 & 3.7261723605 & -1.156 & -2.2134320718 \\
\hline $7 \mathrm{C}$ & 3.7353142270 & -1.0487499041 & -3.5974177221 \\
\hline $8 \mathrm{C}$ & 54 & -0 & -4 \\
\hline $\mathrm{C}$ & 1.9512115208 & 0.6240403271 & -3.5942539704 \\
\hline $0 \mathrm{H}$ & 0.4362588838 & 1.8785715038 & -1.4783882260 \\
\hline $\mathrm{H}$ & 1.4599623189 & 0.4152067896 & 5.14743150 \\
\hline 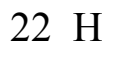 & 1673 & 6651 & 3.2 \\
\hline $3 \mathrm{H}$ & .4007631404 & -1.8336520304 & -1.70 \\
\hline
\end{tabular}




$\begin{array}{lrrr}24 \mathrm{H} & 1.2819142345 & 1.2951031566 & -4.1236452483 \\ 25 \mathrm{H} & -0.0281001749 & 1.7080625618 & 6.6640926536 \\ 26 \mathrm{H} & 2.8933331502 & -0.1150774518 & -5.3685903874 \\ 27 \mathrm{~S} & 4.8967617264 & -2.0407494530 & -4.5465823016 \\ 28 \mathrm{O} & 4.0889015978 & -2.8066329019 & -5.5701868007 \\ 29 \mathrm{O} & 5.7873326503 & -1.0771782688 & -5.2996901301 \\ 30 \mathrm{O} & 5.6217065250 & -2.9015280326 & -3.5821156603 \\ 31 \mathrm{~S} & -2.0777876178 & 3.6975364504 & 6.1822413076 \\ 32 \mathrm{O} & -2.7786127141 & 2.6819535743 & 7.0545288691 \\ 33 \mathrm{O} & -1.1577099170 & 4.5052205003 & 7.0691104846 \\ 34 \mathrm{O} & -2.9900816338 & 4.5169401517 & 5.3519098562 \\ 35 \mathrm{Na} & 5.1958232897 & -1.8867308729 & -7.3588194719 \\ 36 \mathrm{Na} & -1.9028867667 & 3.5245772248 & 9.0061089919 \\ 37 \mathrm{O} & 3.1120749223 & -0.8345151436 & 0.8897101431 \\ 38 \mathrm{H} & 2.6975520922 & -0.5090570373 & 1.7335888806\end{array}$

Indigo, keto $\mathrm{S}_{0}$ :

Coordinates (Angstroms)

\begin{tabular}{|c|c|c|c|}
\hline $\mathrm{OM}$ & $\mathrm{X}$ & Z & \\
\hline $1 \mathrm{~N}$ & 0.0180647699 & -2.7025706523 & 4.0942052069 \\
\hline $2 \mathrm{C}$ & 0.0924600853 & -1.3234735514 & 4.237 \\
\hline $3 \mathrm{C}$ & -0.0283631066 & -3.037 & $2.75^{\circ}$ \\
\hline $4 \mathrm{C}$ & 0.0976029742 & -0.7081777742 & 2.9623772340 \\
\hline $5 \mathrm{C}$ & 0.0210194946 & -1.7727769394 & 1.9635465731 \\
\hline $6 \mathrm{C}$ & -0.1060634088 & -4.3029983804 & 2.2460038847 \\
\hline $7 \mathrm{C}$ & -0.15 & -5.56 & 3.0 \\
\hline $8 \mathrm{C}$ & -0.2320898567 & -6.6324274510 & 2.0409528222 \\
\hline $9 \mathrm{C}$ & -0.2269292001 & -6.0171326123 & 0.7658748725 \\
\hline
\end{tabular}




\begin{tabular}{|c|c|c|c|}
\hline $0 \mathrm{~N}$ & 0.1524819043 & -4.6380381999 & 0.90912 \\
\hline$\Omega$ & -0.0019966564 & -1.7117528177 & 0.7238489694 \\
\hline( & -0.13246 & -5.62 & 4 \\
\hline c & 0.1566084735 & -0.5640216851 & 5.4079615792 \\
\hline 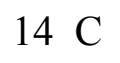 & 0.2261740159 & 0.8251204028 & 5.2689449295 \\
\hline C & 843760 & 1.45 & 4.00 \\
\hline ( & 0.1677342412 & 0.6857117730 & 2.8424823890 \\
\hline 0 & -0.3022883536 & -8.0263134879 & 2.1608491277 \\
\hline $8 \mathrm{C}$ & -0.3667777174 & -8.7924679561 & 0.9 \\
\hline $\mathrm{C}$ & -0.36 & -8.16 & 30351 \\
\hline$C$ & -0.2911191746 & -6.7765823621 & -0. \\
\hline $\mathrm{H}$ & -0.0062341224 & -3.38 & 0655 \\
\hline $2 \mathrm{H}$ & -0.1281855073 & -3.9575416409 & 0.158 \\
\hline $\mathrm{H}$ & 0.1528943915 & -1.0340521163 & 6.3871633071 \\
\hline 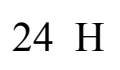 & 0.2872973507 & 2.5347 & 3.94 \\
\hline $5 \mathrm{H}$ & 0.1712839389 & 1.1531619112 & 1.861 \\
\hline $\mathrm{H}$ & -0.3058512296 & -8.4937629148 & 3.1421175075 \\
\hline $7 \mathrm{H}$ & -0.4219376584 & -9.8753425219 & 1.0600792124 \\
\hline $\mathrm{H}$ & -0.2873938784 & -6.3065525433 & -1.3838329825 \\
\hline 1 & 0.2772223633 & 1.4390838190 & 6.1645798940 \\
\hline & -0.4118272852 & -8.7796829619 & -1.1612472735 \\
\hline
\end{tabular}

Indigo, keto $\mathrm{S}_{1}$ :

Coordinates (Angstroms)

$\begin{array}{ccccc}\text { ATOM } & \mathrm{X} & \mathrm{Y} & \mathrm{Z} & \\ 1 \mathrm{~N} & 0.0176487853 & -2.6826702696 & 4.0630021880 \\ 2 \mathrm{C} & 0.0922405525 & -1.3141553932 & 4.2264599416 \\ 3 \mathrm{C} & -0.0275130996 & -3.0121103749 & 2.7271180514\end{array}$




\begin{tabular}{lllll}
$4 \mathrm{C}$ & 0.0969820020 & -0.7049560942 & 2.9409190770 \\
$5 \mathrm{C}$ & 0.0204931981 & -1.7753638946 & 1.9402006644 \\
$6 \mathrm{C}$ & -0.1059650436 & -4.3284612952 & 2.2759882159 \\
$7 \mathrm{C}$ & -0.1543640232 & -5.5651327690 & 3.0629953370 \\
$8 \mathrm{C}$ & -0.2313603163 & -6.6355808532 & 2.0623668758 \\
$9 \mathrm{C}$ & -0.2264804428 & -6.0264867859 & 0.7767913880 \\
$10 \mathrm{~N}$ & -0.1513423403 & -4.6579759125 & 0.9401350401 \\
$11 \mathrm{O}$ & -0.0017566509 & -1.6978776038 & 0.6844304027 \\
$12 \mathrm{O}$ & -0.1320506716 & -5.6425271274 & 4.3187652987 \\
$13 \mathrm{C}$ & 0.1560872986 & -0.5684813774 & 5.40565668880 \\
$14 \mathrm{C}$ & 0.2263346227 & 0.8251596906 & 5.2798932936 \\
$15 \mathrm{C}$ & 0.2318480446 & 1.4450685946 & 4.0165656134 \\
$16 \mathrm{C}$ & 0.1671988408 & 0.6842567433 & 2.8375771122 \\
$17 \mathrm{C}$ & -0.3020755103 & -8.0247562114 & 2.1658635975 \\
$18 \mathrm{C}$ & -0.3671401775 & -8.7856531713 & 0.9869645782 \\
$19 \mathrm{C}$ & -0.3615106263 & -8.1658497092 & -0.2764235334 \\
$20 \mathrm{C}$ & -0.2907252134 & -6.7722519369 & -0.4023288560 \\
$21 \mathrm{H}$ & -0.0063960707 & -3.3815143275 & 4.8029910249 \\
$22 \mathrm{H}$ & -0.1271253795 & -3.9592057997 & 0.2000904942 \\
$23 \mathrm{H}$ & 0.1517001947 & -1.0508465327 & 6.3789428269 \\
$24 \mathrm{H}$ & 0.2869857567 & 2.5281723858 & 3.9531517604 \\
$25 \mathrm{H}$ & 0.1717131752 & 1.1685875044 & 1.8645533960 \\
$27 \mathrm{H}$ & -0.3066612273 & -8.5089817623 & 3.1389384386 \\
$30 \mathrm{H}$ & -0.4226689111 & -9.8687317570 & 1.0504813754 \\
\hline $\mathrm{H}$ & -0.2862404964 & -6.2899815554 & -1.3756619540 \\
\hline & 0.2775108037 & 1.4363215071 & 6.1764892540 \\
\hline
\end{tabular}


Indigo, enol $\mathrm{S}_{1}$ :

Coordinates (Angstroms)

$\begin{array}{ccccc}\text { ATOM } & \mathrm{X} & \mathrm{Y} & \mathrm{Z} & \\ 1 \mathrm{~N} & 0.1154927444 & 1.2592573590 & 1.4312924661 \\ \text { 2 C } & 0.0609665129 & 0.8383819898 & 2.7447796528 \\ \text { 3 C } & 0.0165228459 & 0.1703692136 & 0.5923644741 \\ \text { 4 C } & -0.0790930473 & -0.5851449911 & 2.7600913100 \\ \text { 5 C } & -0.1057030851 & -0.9952673940 & 1.4025645911 \\ \text { 6 C } & 0.0338322859 & 0.2068065434 & -0.8155738775 \\ \text { 7 C } & 0.1578008991 & 1.3664925912 & -1.7003663977 \\ \text { 8 C } & 0.1121043648 & 0.7630757769 & -3.0399244701 \\ \text { 9 C } & -0.0304269977 & -0.6544774635 & -2.8314345357 \\ 10 \mathrm{~N} & -0.0759687985 & -0.9725151321 & -1.5060689571 \\ 11 \mathrm{O} & -0.2237364523 & -2.2499087181 & 0.9549831648 \\ 12 \mathrm{O} & 0.2740361758 & 2.5744976579 & -1.3584297021 \\ 13 \mathrm{C} & 0.1218094485 & 1.5780229207 & 3.9263220139 \\ 14 \mathrm{C} & 0.0404089575 & 0.8741096863 & 5.1315060850 \\ 15 \mathrm{C} & -0.0983366592 & -0.5330959993 & 5.1661868669 \\ 16 \mathrm{C} & -0.1590669098 & -1.2724362618 & 3.9908726298 \\ 17 \mathrm{C} & 0.1771429611 & 1.2775635866 & -4.3264569229 \\ 18 \mathrm{C} & 0.1005068337 & 0.3801144170 & -5.4102881971 \\ 19 \mathrm{C} & -0.0392933574 & -1.0111371866 & -5.2078010190 \\ \text { 20 C } & -0.1065079669 & -1.5454944085 & -3.9223419246 \\ \text { 21 H } & 0.2114711069 & 2.2140147443 & 1.1036046472 \\ \text { 22 H } & 0.2281542929 & 2.6588588128 & 3.9092555626 \\ \text { 23 H } & -0.1577531232 & -1.0382790898 & 6.1259889530\end{array}$




$\begin{array}{llll}24 \mathrm{H} & -0.2654596633 & -2.3533197393 & 4.0132675070 \\ 25 \mathrm{H} & 0.2845621856 & 2.3450293052 & -4.5024569570 \\ 26 \mathrm{H} & 0.1495529787 & 0.7654403958 & -6.4250776768 \\ 27 \mathrm{H} & -0.2137956421 & -2.6137613213 & -3.7549024169 \\ 28 \mathrm{H} & -0.2100107967 & -2.2120734412 & -0.0393150406 \\ 29 \mathrm{H} & 0.0852168990 & 1.4252288861 & 6.0670623277 \\ 30 \mathrm{H} & -0.0948530938 & -1.6707508400 & -6.0690078567\end{array}$

Indigo, enol $\mathrm{S}_{1}$ via SF-TDDFT:

Coordinates (Angstroms)

$\begin{array}{lccc}\text { ATOM } & \mathrm{X} & \mathrm{Y} & \mathrm{Z} \\ \text { 1 N } & 0.1093038956 & 1.2029957095 & 1.4117425778 \\ \text { 2 C } & 0.0589439498 & 0.8157171937 & 2.7247138000 \\ \text { 3 C } & 0.0108761999 & 0.1185846677 & 0.6030064597 \\ \text { 4 C } & -0.0794151411 & -0.5919054483 & 2.7586492805 \\ \text { 5 C } & -0.1077920397 & -1.0205809162 & 1.4056154167 \\ \text { 6 C } & 0.0301306008 & 0.1746344666 & -0.8294700613 \\ \text { 7 C } & 0.1539870678 & 1.3395380712 & -1.6874549031 \\ \text { 8 C } & 0.1109498738 & 0.7587482549 & -3.0249691996 \\ \text { 9 C } & -0.0299932945 & -0.6395801903 & -2.8374392875 \\ 10 \mathrm{~N} & -0.0759246068 & -0.9636232159 & -1.5008516198 \\ 11 \mathrm{O} & -0.2243705316 & -2.2729427660 & 0.9931499946 \\ 12 \mathrm{O} & 0.2672810220 & 2.5298727931 & -1.3194098140 \\ 13 \mathrm{C} & 0.1223784724 & 1.5754641852 & 3.8823373895 \\ 14 \mathrm{C} & 0.0436106405 & 0.8969721910 & 5.0916577462 \\ 15 \mathrm{C} & -0.0945646856 & -0.4956584677 & 5.1435822290 \\ 16 \mathrm{C} & -0.1569378824 & -1.2532315160 & 3.9839996307 \\ 17 \mathrm{C} & 0.1786307118 & 1.2859227264 & -4.3008124004\end{array}$




$\begin{array}{lllll}18 \mathrm{C} & 0.1042106412 & 0.4062690162 & -5.3837947409 \\ 19 \mathrm{C} & -0.0355592788 & -0.9713832820 & -5.1945584932 \\ 20 \mathrm{C} & -0.1046024644 & -1.5129221180 & -3.9152002527 \\ 21 \mathrm{H} & 0.2020007802 & 2.1440511667 & 1.0642852235 \\ 22 \mathrm{H} & 0.2285245996 & 2.6470695141 & 3.8442266780 \\ 23 \mathrm{H} & -0.1527672207 & -0.9846073901 & 6.1020096467 \\ 24 \mathrm{H} & -0.2628644749 & -2.3246872789 & 4.0278975950 \\ 25 \mathrm{H} & 0.2864982206 & 2.3467381707 & -4.4627141148 \\ 26 \mathrm{H} & 0.1546591918 & 0.7975155447 & -6.3872621973 \\ 27 \mathrm{H} & -0.2124063657 & -2.5743524295 & -3.7590786321 \\ 28 \mathrm{H} & -0.2150897004 & -2.2803374044 & 0.0179826760 \\ 29 \mathrm{H} & 0.0901979491 & 1.4569780431 & 6.0111954804 \\ 30 \mathrm{H} & -0.0903202309 & -1.6216573916 & -6.0523398076\end{array}$

Indigo, MECP via SF-TDDFT:

Coordinates (Angstroms)

$\begin{array}{ccccc}\text { ATOM } & \text { X } & \text { Y } & Z & \\ 1 \mathrm{~N} & 0.1177377222 & 1.2644790387 & 1.3940849532 \\ 2 \mathrm{C} & 0.0635555274 & 0.8486427526 & 2.6800449644 \\ 3 \mathrm{C} & 0.0172346234 & 0.1689130678 & 0.5576247602 \\ 4 \mathrm{C} & -0.0758437409 & -0.5634610710 & 2.7096887864 \\ \text { 5 C } & -0.1040746689 & -0.9883141487 & 1.3692408380 \\ \text { 6 C } & 0.0351183524 & 0.2312817648 & -0.8105584308 \\ 7 \mathrm{C} & 0.1513979747 & 1.3387258041 & -1.6287570861 \\ \text { 8 C } & 0.1080828079 & 0.7490580223 & -2.9444002684 \\ \text { 9 C } & -0.0414755060 & -0.7400038357 & -2.7559684902 \\ 10 \mathrm{~N} & -0.0889469276 & -1.0903332222 & -1.5509458454 \\ 11 \mathrm{O} & -0.2208788361 & -2.2358329505 & 0.9631194889\end{array}$




$\begin{array}{lllll}12 \mathrm{O} & 0.2686247267 & 2.5679836886 & -1.2769462291 \\ 13 \mathrm{C} & 0.1241289130 & 1.5883238281 & 3.8649685187 \\ 14 \mathrm{C} & 0.0433927738 & 0.8925095295 & 5.0486832473 \\ 15 \mathrm{C} & -0.0959504010 & -0.5155450160 & 5.0935437416 \\ 16 \mathrm{C} & -0.1557254899 & -1.2479933479 & 3.9372840889 \\ 17 \mathrm{C} & 0.1775732769 & 1.2844190672 & -4.2140173815 \\ 18 \mathrm{C} & 0.1064446533 & 0.4311106505 & -5.2839746210 \\ 19 \mathrm{C} & -0.0403603646 & -1.0213352417 & -5.1414603390 \\ 20 \mathrm{C} & -0.1132777780 & -1.6017146559 & -3.9483098438 \\ 21 \mathrm{H} & 0.2089743254 & 2.2025448302 & 1.0432986448 \\ 22 \mathrm{H} & 0.2300159621 & 2.6601676772 & 3.8442195574 \\ 23 \mathrm{H} & -0.1544175382 & -1.0064567605 & 6.0508125713 \\ 24 \mathrm{H} & -0.2612983946 & -2.3203015323 & 3.9577294041 \\ 25 \mathrm{H} & 0.2853195603 & 2.3465561900 & -4.3624611838 \\ 26 \mathrm{H} & 0.1580245461 & 0.8271897821 & -6.2845061209 \\ 27 \mathrm{H} & -0.2213385019 & -2.6654420387 & -3.8176365042 \\ 28 \mathrm{H} & -0.2123487124 & -2.2477488018 & -0.0163192822 \\ 29 \mathrm{H} & 0.0878470263 & 1.4378570503 & 5.9777759767 \\ 30 \mathrm{H} & -0.0879600126 & -1.6056782214 & -6.0451616151\end{array}$

\section{REFERENCES}

(1) Oliver, T. A. A.; Lewis, N. H. C.; Fleming, G. R. Correlating the Motion of Electrons and Nuclei with Two-Dimensional Electronic-Vibrational Spectroscopy. Proc. Natl. Acad. Sci. USA 2014, 111, 10061-10066. https://doi.org/10.1073/pnas.1409207111. 